\title{
tic\&société
}

Vol. 9, No 1-2 | 1 er semestre 2015 - 2ème semestre 2015

Les religions au temps du numérique

\section{Cérémonies de mariage dans World of Warcraft : transfert rituel ou institution collective?}

\section{Olivier SERVAIS}

\section{(2) OpenEdition \\ Journals}

\section{Electronic version}

URL: http://journals.openedition.org/ticetsociete/1823

DOI: 10.4000/ticetsociete.1823

\section{Publisher}

Association ARTIC

\section{Electronic reference}

Olivier SERVAIS, «Cérémonies de mariage dans World of Warcraft:

transfert rituel ou institution collective? », tic\&société [Online], Vol. 9, № 1-2 | 1er semestre 2015 -

2ème semestre 2015, Online since 08 January 2016, connection on 19 April 2019. URL : http://

journals.openedition.org/ticetsociete/1823; DOI : 10.4000/ticetsociete.1823 
tic\&société - 9 (1-2), 2015

\section{Cérémonies de mariage dans World of Warcraft : Transfert rituel ou institution collective?}

Olivier SERVAIS

Laboratoire d'anthropologie prospective - Institut lacchos

Université catholique de Louvain, Place Montesquieu, 1/1

bte L2.08.01, 1348-Louvain-La-Neuve, Belgique

olivier.servais@uclouvain.be 
Cérémonies de mariage dans World of Warcraft

\section{Olivier SERVAIS}

\section{Cérémonies de mariage dans World of Warcraft : Transfert rituel ou institution politico-religieuse ?}

Olivier SERVAIS est professeur à l'Université de Louvain (Louvainla-Neuve) en Belgique, où il enseigne l'anthropologie des systèmes symboliques, des religions et des environnements naturels et numériques. Ses intérêts de recherche sont actuellement centrés sur les jeux vidéos, les mondes virtuels et les religions et leurs relations aux environnements technologiques. II a coordonné ou participé à plusieurs projets de recherche multidisciplinaires incluant la collecte et l'analyse de données tant qualitatives que quantitatives en Belgique, en France, au Canada et dans les mondes virtuels.

Résumé : Mes recherches des trois dernières années ont été axées notamment sur la religion en ligne dans l'univers virtuel du monde du jeu vidéo of World of Warcraft (WoW) dans sa réalité de langue française. Un premier moment donnera un bref aperçu des différents points de vue de la recherche actuelle sur l'étude de la religion sur l'internet, cyber-ritualité et WoW. Cette section préliminaire servira en partie de cadre de l'enquête. Deuxièmement, nous allons concentrer notre discussion sur une question importante de cette transformation religieuse, le cas du mariage cyber dans WoW. À partir de 100 cérémonies, nous allons analyser des rituels de mariage, fêtes via avatars (représentations numériques des individus), où les guildes (les communautés de joueurs) développent analogiquement une fonction politicoreligieuse. Enfin, nous allons examiner les changements socioanthropologiques que cette affaire révèle et les questions théoriques connectées. 


\section{Olivier SERVAIS}

Mots-clés : Cyber-mariage, World of Warcraft, rituel, religion en ligne, ethnographie.

Abstract: Wedding Ceremonies in World of Warcraft: Ritual transfer or collective institution? My research over the past three years has been focused particularly on online religion in the virtual universe of the videogame World of Warcraft (WoW) as it is played in French. A first moment will give a brief outline of the different perspectives in current research on the study of religion on the Internet, cyber-rituality and WoW. This preliminary part will thus serve as part of the framework of the investigation. Secondly, we will focus our discussion on a significant question about this religious transformation, the case of cyber marriage in WoW. Using a corpus of 100 ceremonies we will analyse wedding rituals, celebrations via avatars (digital representations of individuals), in which guilds (player's communities) develop a politico-religious function analogically. Finally, we will consider the socioanthropological changes that this case reveals, and realted theoretical questions.

Keywords: Cyber-weddings, World of Warcraft, Ritual, Online religion, Ethnography.

Resumen: Ceremonias de matrimonio en World of Warcraft: Transferencia Ritual o institución colectiva? Mi investigación en los últimos tres años se ha centrado sobre todo en la religión en el mundo virtual del videojuego World of Warcraft (WoW), en lengua francesa. Una primera parte dará una breve descripción de las diferentes perspectivas de las investigaciones actuales sobre el estudio de la religión en el Internet, el ritual cibernética y WoW. Esta parte preliminar servirá como marco de la investigación. En segundo lugar, centraremos nuestra discusión sobre un tema importante de esta transformación religiosa,: el caso del cyber matrimonio en WoW. Considerando 100 ceremonias, se analizan los rituales de la boda, las fiestas a través de avatares (representaciones digitales de los individuos), en donde los 


\section{Cérémonies de mariage dans World of Warcraft}

gremios (las comunidades de jugadores) desarrollan de forma análoga una función político-religiosa. Por último, se analizan los cambios socio-antropológicos que este caso pone de manifiesto, y las cuestiones teóricas que suscitan.

Palabras clave: Cyber Matrimonio, World of Warcraft, Ritual, Cyber Religión, Etnografía.

Mes recherches de ces trois dernières années ont porté en particulier sur la religion dans les univers virtuels (Servais, 2012, 2013) et plus spécifiquement, dans divers jeux en ligne dont World of Warcraft (WoW) ${ }^{1}$. Or, cet univers de WoW assume une position singulière. Depuis 2004, il représente un des jeux vidéos en ligne de référence tant en terme de nombre d'abonnés que de présence médiatique. $\mathrm{Ce}$ type de monde 3D est particulièrement représentatif de ces nouveaux jeux massivement multi-joueurs, ayant intégrés en leur cœur la dimension sociale à côté de la finalité ludique. Si le joueur incarne un personnage, un avatar, les guildes, ces collectifs de référence dans le jeu, prennent une place centrale (Brignall, 2008). Ces micro-groupes sociaux de quelques dizaines de personnages sont la clé de la réussite du jeu (Ducheneaut et al., 2006). De fait, on pratique rarement avec des groupes de très grandes tailles (Williams et al., 2006). Les abonnés sont répartis par serveurs (appelés royaumes) sur une base linguistique. Dans sa forme originale, la scénographie y est principalement médiévale-fantastique, de type jeu de rôle classique, ancrée dans un environnement dosant habilement le compétitif et le collaboratif et structurée autour de deux factions opposées, l'Alliance et la Horde. Les joueurs peuvent s'affronter entre factions (Players versus Players, dit $P \vee P$ ) ou affronter ensemble des créatures mues par l'ordinateur (Players versus Environment, dit $P v E$ ), chaque royaume étant régi par l'un de ces deux modes de jeu. S'ajoute à cette distinction binaire un mode dit " jeu de rôle " sur certains serveurs dont les membres choisissent d'incarner plus en profondeur leur personnage. Le style de jeu est tourné vers la performance d'acteur des joueurs. Si la montée en

${ }^{1}$ World of Warcraft [Computer Software]. Irvine, CA : Blizzard Entertainment 


\section{Olivier SERVAIS}

puissance des personnages débute souvent seul, à partir d'un certain niveau les joueurs autonomes sont par la force des choses contraints de rejoindre une guilde. Ces regroupements plus ou moins soudés varient d'une dizaine à un millier de joueurs.

À partir d'une immersion ethnographique de trois années (avril 2010 à avril 2013) au cœur de WoW, à l'image d'une Bonnie Nardi (Nardi, 2010), j'ai exploré ce métavers ${ }^{2}$. Les deux premières années furent celle d'une immersion intense au sein d'une même guilde de joueurs de l'Alliance. L'observation s'est déroulée sur le serveur francophone Varimathras, un serveur PVE à raison d'au moins 6 à 8 h par jour.

Je me suis alors focalisé progressivement et, à partir d'une épistémologie hypothético-inductive, sur un certain nombre de problématiques émergeant du terrain, dont celle des cérémonies. Pour ce faire, je me suis inspiré de Taylor (2006) et d'une socioanthropologie des appropriations par les joueurs de leur environnement numérique, ou de Boellstorff (2008) concernant l'émergence d'une culture propre à ces métavers. J'ai ainsi tenté d'interpréter les bricolages rituels des usagers. J'ai décidé de partir du point de vue émique, celui des acteurs, c'est-à-dire d'observer, de répertorier et d'analyser ce que les joueurs/pratiquants euxmêmes désignaient par «mariage » dans le jeu.

Suivant la désormais classique typologie de Boellstorff (2009), qui distingue trois approches pour l'ethnographie du virtuel virtuel / virtuel, virtuel / actuel et virtuel dans leurs propres termes -, je choisis d'étudier dans le cas du présent travail les cyber-mariages « in their own terms ». Il y aurait un grand intérêt à s'arrêter sur l'articulation virtuel/actuel concernant les mariages ou sur une comparaison selon les différents métavers. Mon propos se limitera ici aux cérémonies en ligne qualifiées de mariages dans le WoW francophone. Au-delà des contraintes limitées de l'article, une raison plus fondamentale m'y pousse. Même si on croit référer à la même chose à travers le terme "mariage ", il n'est pas

\footnotetext{
${ }^{2}$ Le terme « métavers » provient de l'anglais metaverse, contraction de meta universe, et qualifie un monde virtuel fictif ; le mot a été utilisé abondamment dans la littérature pour définir les mondes sociaux numériques en trois dimensions.
}

tic\&société - 9 (1-2), 2015 


\section{Cérémonies de mariage dans World of Warcraft}

impossible qu'entre mariages actuels et mariages virtuels, on ait affaire à des réalités partiellement différentes. Car même s'ils en partagent la dénomination et certaines formes, mariage physique et mariage numérique n'en sont pas moins conditionnés par des contextes et des indexicalités essentiellement autres qui nécessiteraient une enquête auprès de tous les joueurs pour connaître leur rapport personnel au mariage et leur condition maritale personnelle. Une telle investigation nous emmènerait trop loin pour le présent article.

Un premier moment rapide donnera un bref aperçu des différentes perspectives de la recherche actuelle sur l'étude des rituels religieux sur Internet dont les cyber-mariages. Dans un second temps, je concentrerai mon propos sur les apports d'une ethnographie des mariages dans WoW. Enfin, à partir des données synthétisées, je montrerai de manière embryonnaire en quoi ces cérémonies vidéo-ludiques questionnent certaines théories sur la pratique rituelle en ligne ${ }^{3}$.

\section{WoW, le religieux et le rituel}

Le monde de Warcraft est un univers pétri d'imaginaire religieux. Ainsi en est-il, par exemple, quand vous mourez, et dans ce jeu cette situation est excessivement fréquente, un ange, agent informatique, vous ressuscite. Nous sommes bien là en présence d'un symbole morphologiquement très religieux. Autre illustration, vous avez plusieurs personnages qui sont apparentés à des figures religieuses: le paladin, le druide, le shaman, le prêtre et dernièrement le moine. Tous ces personnages ont notamment la faculté de guérir les autres joueurs. Une dimension altruiste a donc

3 Je souhaite associer à ce papier les participants des GSALF 2013 à Lyon, de la Conférence de l'International Society for Sociology of Religion à Turku en 2013 et du séminaire doctoral du Cherpa à l'IEP d'Aix en Provence en mars 2014. Par ailleurs, je remercie le FRS-FNRS qui a financé une partie de ma récolte de données. J'associe à cette étape de recueil mes étudiants de $3^{\text {ème }}$ année en sciences humaines et sociales (2013-2014) et Pauline Christians qui m'a assisté dans certains dépouillements. Enfin, je souhaiterais remercier Mike Singleton et Tom Boellstorff pour leurs suggestions d'améliorations. 


\section{Olivier SERVAIS}

été attribuée aux capacités de ces figures religieuses. Nombre de décors sont eux aussi de manière très prégnante à morphologie religieuse : des édifices qui, même s'ils n'arborent pas de croix, de croissants, de statues de Bouddha ou de chandeliers à sept branches, s'apparentent, par leur forme, leur architecture ou leur nom à des édifices religieux. II en est de même pour les cimetières où vous ne trouverez jamais de signes explicites, mais la morphologie religieuse y est patente. Le religieux paraît dans la référence culturelle qu'induit une forme spécifique de bâtiment ou de construction numérique. II en est de même de multiples activités, voire cérémonies, intégrées à la narration du jeu. Cette dimension interne au jeu (game design) et les analyses qui conçoivent le religieux avant tout comme un décor spatial et une mythographie ludique, ont été étudiés par Bainbridge qui y consacre d'abord un plein chapitre (Bainbridge, 2010, pp.53-79) puis un livre (Bainbridge, 2013). Mais l'enquête nous emmène plus loin.

Au-delà d'une dimension religieuse interne à la conception de l'univers de Warcraft, on note la présence et la revendication par certains pratiquants de cérémonies, voire de ritualités, spirituelles ou religieuses, ce que d'aucuns ont appelé des online rituals (Heidbrink, 2007), des cérémonies crées par les joueurs et accomplies par ceux-ci au sein du jeu. J'ai assisté à plusieurs de ces évènements dans Azeroth, les terres de WoW. II s'agit de célébrations que les joueurs ont élaborées entre eux, en dehors des buts du jeu, et qu'ils vivent collectivement via leur avatar. On assiste ainsi tantôt à une cérémonie entre deux joueurs d'une même guilde, qu'ils appellent "un mariage ", tantôt à un hommage collectif à un joueur décédé (Servais, 2012), à la mise en scène de la création d'une guilde, ou encore à la simple célébration par sa guilde du départ du jeu d'un membre. Certaines de ces activités revêtent implicitement pour un nombre de praticiens un caractère différent, peut-être sacré (Dufour et Boutaud, 2013), qu'on peut dès lors apparenter à des rituels "religieux». Sur ce dernier adjectif, je m'en tiendrai aux affirmations prudentes d'Albert Piette sur la nature provisoire, hybride et enchevêtrée de l'attribution de «religieux » (Piette, 2003, p.101). J'ai parlé ailleurs d'un religieux analogique pour 


\section{Cérémonies de mariage dans World of Warcraft}

parler de ces phénomènes de cérémonies virtuelles (Servais, 2013, pp.104-108), même si mon usage de l'analogie est limité : "Les analogies de ce type ne visent pas une théorie d'Internet mais une anthropologie fondée avant tout sur une ethnographie, une description empiriquement fondée et dont l'analogie vient simplement éclairer l'interprétation qui en est produite ».

\section{Cyber mariages, transfert rituel et bricolage}

Au sein des rituels évoqués, les mariages occupent une place de choix. Bien entendu, comme le note Tom Boellstorff, la plupart des univers virtuels fonctionnent d'abord sur la base d'un individualisme des avatars, où la relation d'amitié inter-avatars est la référence première des rapports sociaux (Boellstorff, 2013, pp.328 sq.). Dans Second Life toutefois, le statut de partenaire a ouvert la porte à la relation maritale in Game. C'est d'ailleurs dans cet univers que les relations conjugales numériques ont été probablement les plus étudiées.

Depuis lors, la littérature foisonne sur les cyber-mariages. Klink dans un mémoire de maîtrise en 2008, signalait : "Weddings are not an unusual occurrence in Second Life. Frequently, if both parts of a married couple are both active in Second Life they have a ceremony there to make it "official" in both First and Second Life; sometimes, however, these marriages are between avatars and not the people behind them 》 (Klink, 2008).

Mais la question des cérémonies de mariages virtuels est ancienne. En 1999, Reid évoque ainsi l'existence de telles pratiques dans plusieurs univers numériques dans les années 1990 (Reid, 1999, pp.107-133). En 2007, des spécialistes de la société chinoise constatent un fort développement de cette pratique en lien avec le succès culturel des jeux de rôle en ligne (Weihua et al., 2007), marque d'un phénomène d'expression cachée dans une Chine postsocialiste : ils y voient l'expression d'une sous-culture de jeunes rebelles pratiquant le collage symbolique et l'amalgame pour à la fois démystifier le mariage traditionnel et ouvrir les possibles grâce aux technologies. 


\section{Olivier SERVAIS}

En Occident, à part les travaux de Radde-Antweiler (2007, 2010), pour Second Life, les données sont plus limitées. Souvent c'est l'anecdote qui domine avec une mention courte. Ou alors le rite de mariage est annexé aux autres rituels, oblitérés et dilués dans le tout du rituel. En tout état de cause le mariage a été rarement analysé en profondeur ou sur des échantillons très limités (Shim, 2010). En 2010, William Bainbridge dans son ouvrage, Warcraft Civilization, exemplifie son propos sur l'identité dans le jeu à partir de deux cas de mariage (Bainbridge, 2010, pp.192-197). Mais la cérémonie ne vise au fond qu'à démontrer sa thèse sur le caractère fictif de l'identité : "These two mock weddings, with their rather different relationships between character and player, illustrate the ambiguities of identity in WoW, and the fact that here as elsewhere relations between people are a prime determinant of self. The character of a person, like those avatars WoW calls characters, is a social construction. From the dramaturgical perspective, and perhaps in truth, everybody is always a fictional character. 》(Bainbridge, 2010, p.197).

Perkins signale ainsi en 2011: "During my time in World of Warcraft, I noticed an in-game funeral for a deceased player. This event was held at the largest church in the capitol city and looked like a very grand state funeral with hundreds of attendees. Similar events occur when characters decide to get married. Although there is no in-game functionality for marriage, some players choose to recognize their characters are married." (Perkins, 2011). Ces évocations s'arrêtent souvent là et leurs propos ne sont pas centrés sur la question de la cérémonie de mariage en ellemême, ni sur la portée spécifique de cette dernière.

Dans le monde francophone, Vincent Berry a été plus loin. Dans son dernier livre, L'Expérience virtuelle, il est un des seuls à montrer la complexité des cérémonies de mariage dans WoW (Berry, 2012, pp.162-164). Cette mention prend place dans son chapitre sur les expériences identitaires. Ses trois pages évoquent la densité de cette problématique (articulation des contextes virtuel et actuel ; le style roleplay ou non). Mais, il ne fait qu'illustrer ici encore son propos sur le jouer à partir du mariage qu'il qualifie de faire-semblant. Dans un article antérieur (Berry, 2007), le chercheur soulignait, à travers une image, la célébration d'un 


\section{Cérémonies de mariage dans World of Warcraft}

mariage entre deux joueurs pour sceller «l'union » de deux guildes. Mais ici aussi, le mariage ne fait qu'éclairer les relations de guildes.

Selon Radde-Antweiler (2007), on repère une trame narrative similaire à tous ces rituels de mariages. On peut la résumer de cette façon. Après l'arrivée des invités, la « remise » de la mariée et les premiers mots de l'officiant (appelé souvent « révérend »), et qui est souvent le concepteur du mariage lui-même, la cérémonie commence avec l'interrogation du couple sur différentes questions: s'ils sont disposés à s'aimer l'un l'autre, à être honnêtes et à être présents pour le partenaire, etc. La structure et la forme des mariages sont relativement flexibles, en fonction du genre de mariage ou de cérémonie que le couple préfère. De manière générique, il comprend les auditions des mariés, les vœux, l'échange des alliances et une longue auto-déclaration écrite au sujet de leur amour et de leur engagement à l'autre. La reproduction explicite de traditions religieuses est l'exception plutôt que la norme, mais il est possible d'identifier des processus implicites de réception et la combinaison d'éléments issus de différentes traditions, comme des éléments de la liturgie anglicane mélangés à des éléments de rituels contemporains culturellement dominants.

La théorisation la plus aboutie est l'œuvre du groupe de recherche dit d'Heidelberg explorant le religieux dans les mondes virtuels (Langer et al., 2006) et où la ritualité dans les cyberespaces, et singulièrement les mariages y occupent une position centrale (Radde-Antweiler, 2007 ; 2010). Radde-Antweiler y voit un cas emblématique de transfert rituel du monde actuel vers le monde virtuel. Cette théorie reconnaît que les rituels sont dynamiques et changeants et tente d'identifier les différents éléments, internes ou contextuels, altérés ou transformés quand un rituel est transféré vers un nouveau media (Langer, 2006, pp.24 ; Helland, 2013, p.35). Sont retenues trois dimensions caractéristiques de ce transfert: la transformation sous de multiples formes du rituel, l'invention de nouveaux éléments relatifs au nouvel univers de réception et enfin l'exclusion de parties du rituel originel (Miczek, 2008). Le rite transféré, malgré ses transformations, doit alors faire l'objet d'une reconnaissance d'authenticité par les praticiens pour être jugé équivalent. 


\section{Olivier SERVAIS}

La théorie du transfert rituel a le grand mérite de tenter une conceptualisation afin d'appréhender les contraintes des ritualités en ligne. Elle montre aussi quelques limites, en ne reliant pas son cadre heuristique aux théories classiques du syncrétisme, du métissage ou du bricolage religieux (Christians, Servais, 2005). En outre, quelles que soient ces perspectives, elles se focalisent énormément sur le rituel en lui-même. Et en se centrant sur le rite, elle le fait exister. Or un rite est souvent bien autre chose qu'un rite, notamment du point de vue des protagonistes.

Les auteurs évoquent très peu ce que Houseman et Severi (1998) appellent le champ relationnel du rite ${ }^{4}$. Or, "l'efficacité rituelle se rapporte en premier lieu aux changements relationnels que permet de médiatiser ce processus : elle consiste moins en la communication de messages à déchiffrer - des "textes" - qu'en la transmission de contextes singuliers et hautement intégratifs favorisant des discours et des actions intervenant au-delà du rite, mais qui présupposent les relations réalisées au cours de sa performance. » (Houseman 2008, p.1). Je souhaiterais coupler cette logique de performativité relationnelle et contextuelle à la fonction sociale du rite que je qualifierai maintenant de "religieux » au sens de Godelier (2007). Celui-ci défend l'idée que le «politico-religieux » fonde la société. Pour faire une société, ni la parenté ni les liens de production et d'échange de biens ne sont suffisants dit-il. II faut surtout que des croyances religieuses et des rituels qui les mettent en actes viennent légitimer une souveraineté (le politique) et assurer sa reproduction sur un territoire donné. Ce qui confère une identité sociale globale aux individus comme aux groupes, ce sont donc les rapports politico-religieux au sein desquels les individus et les groupes se trouvent placés et qu'ils doivent reproduire et transformer. II balise le mariage à partir du pouvoir et affirme qu'il a pour fonction la transmission des " rapports politico-religieux » entre générations. Concrètement, le mariage, ou toute institution fonctionnellement similaire, permet aux hommes de transmettre l'ordre social aux enfants qu'ils auront engendrés et de ce fait permettra à la société de se reproduire.

\footnotetext{
${ }^{4} \mathrm{Si}$ ce n'est pour citer trois paragraphes sur les postures des participants (Langer et al., p.3).

tic\&société - 9 (1-2), 2015 
Cérémonies de mariage dans World of Warcraft

À partir de ces deux perspectives théoriques, l'une centrée sur le rite et ses caractéristiques (notamment transférées) et l'autre sur sa performance relationnelle fondant une société, ici virtuelle, il me semblait pertinent de revisiter mon ethnographie.

\section{Faire l'ethnographie en ligne de mariages dans World of Warcraft}

Après six mois dans la guilde des Dragons Immortels, j'avais eu l'occasion d'échanger avec une large partie des habitants de la collectivité (j'appelle habitants les joueurs très actifs). Dans un entretien avec l'un des officiers, un prêtre nain, celui-ci m'expliquait : " Mon avatar est nommé "Peremaiter", mais en fait, tout le monde m'appelle "Padre" (« mon Père »)... Un jour, deux persos sont venus me demander si je pouvais célébrer leur mariage! Ils étaient ensemble dans le jeu, mais aussi dans la réalité, ils voulaient vraiment se marier devant leurs amis virtuels... "Peremaiter" est un prêtre, c'est pourquoi ils me l'ont demandé. J'ai accepté et créé une cérémonie basée sur la "religion pomme". La bien-aimée était elfe de sorte que ça avait un sens pour moi. J'ai préparé le rituel avec les macros et autres ressources disponibles dans WoW et nous avons célébré le mariage avec beaucoup de leurs amis... 》 (Entretien avec Padre, avril 2012).

Étant donné mon intérêt pour les rituels et leur bricolage, mon attention s'est trouvée décuplée par cette histoire. J'avais bien entendu constaté depuis longtemps que le dispositif technique du jeu n'inclut pas le statut de marié, contrairement à d'autres univers. Le mariage est donc de l'ordre du détournement du jeu par les joueurs, au sens du braconnage de Certeau (1990).

En recherchant sur les différents forums du serveur, je ne trouvais d'ailleurs pas plus d'allusions à ce rituel de la pomme inventé par Padre. Sur Judge Hype je repérais la seule mention proche: Malacath avait posté le commentaire suivant le 23-092007: " J'avais entendu parler de frutimariages à une époque. C'était une cérémonie fruitée avec un frutiprêtre ". C'était bien 


\section{Olivier SERVAIS}

entendu trop peu pour continuer. Mais j'avais l'envie de poursuivre à investiguer cette question.

De forums de guildes en sites de joueurs, j'ai alors méticuleusement recherché tous les mariages s'étant déroulés sur Varimathras, le serveur où je menais mon observation. C'est à ce moment que je suis tombé sur une vidéo, intitulée Mariage de Niloweana et de Skïdda ${ }^{5}$ qui venait de se dérouler en mai 2011. Ce film suscitait beaucoup de réactions sur la toile, 62 au total. La présentation décrivait la vidéo comme suit : "Voici mon mariage avec mon chéri Skïdda. II se déroule au Temple de la Lune à Darnassus. Sur le Royaume Varimathras. Merci aux gens présents ce jour-là. Gros merci à Saïko qui a exercé son rôle à $100 \%$, et à Hiraïko qui s'est démené à fond pour son "stuff" à l'occasion. Je tiens à remercier personnellement Draënauzor, le réalisateur, à qui j'en ai fait baver $=)$. »

Après une analyse approfondie du film, j'examinais aussi en détail les commentaires. Ces données intégrées nous donnent une trame d'un mariage sur WoW. La vidéo me permet de décrire brièvement cette première cérémonie. II s'agit du mariage de deux Draenei à Darnassus. Le lieu a été choisi par les futurs époux. Le film commence avec l'arrivée des conjoints accompagnés de leurs témoins dont un qui filme la scène. La mariée est en blanc "comme il se doit » et le marié en costume. Ensuite, une fois entrés dans le temple d'Elune, on assiste à une célébration par une prêtresse, Saîko, habillée de noir. Manifestement, celle-ci nous livre une longue narration en lien avec les Naaru et la mythologie Draenei. Cependant la qualité de la vidéo ne nous donne pas accès aux détails du discours. Au total, 8 personnagesjoueurs sont présents: une célébrante, 2 futurs-mariés et 5 assistants. Après l'échange des consentements, on lance les feux d'artifice et des cônes de lumière, et enfin une fête s'organise sur place avec danses collectives et exercice de « striptease » dans le temple.

\footnotetext{
${ }^{5}<$ http://worldofwarcraft.judgehype.com/news/video-mariage-de-niloweana-et-de-skiddasur-varimathras/>, dernière consultation le 16 avril 2015 ; on la trouve aussi sur Youtube : <http://www.youtube.com/watch?v=Jdr29cwyRaM>, mise en ligne le 17 mai 2011 (dernière consultation le 16 avril 2015).

tic\&société - 9 (1-2), 2015 


\section{Cérémonies de mariage dans World of Warcraft}

À côté de l'observation permise par la caméra, les commentaires m'ont autorisé à en comprendre un peu plus sur le contexte de cet évènement, et cela d'autant que la mariée, Niloweana y est intervenue. En effet, un nombre important de joueurs s'indigna qu'une telle vidéo soit déposée sur un site consacré aux jeux vidéos. Le débat qui s'ensuivit fut parfois assez rude. Parmi les éléments mis en évidence dans ces données de forum on retiendra: la qualité du discours de l'officiante, ou des éléments sur l'articulation mariage en ligne et situation hors ligne des conjoints. Cependant, la dimension polémique des discussions et la qualité relativement faible de la vidéo ne m'a pas permis d'en savoir beaucoup plus. Ces investigations préliminaires m'ont toutefois aidé à découvrir la problématique et à en mesurer une part des dimensions structurantes. La nature houleuse des questions sur ces cyber-mariages m'a toutefois convaincu que pour aller plus avant sur ces rituels et leur fonction, une analyse des cérémonies virtuelles en elles-mêmes apporterait sans doute des données plus précises, et surtout, je l'espérais, plus nombreuses.

Une fois cette étape préliminaire franchie, et les choix de perspectives adoptés, l'objectif fut donc de changer d'échelle d'analyse et de multiplier les données à observer. Cette visée s'est traduite par une recherche de mariages disponibles sur support vidéo numérique et qui me donneraient accès à une forme d'observation. J'étais bien entendu conscient des limites d'une telle approche : j'aurais accès uniquement aux extraits vidéo ou diaporamas des cérémonies en complément de quelques-unes observées directement sur le terrain. M'échapperaient donc toutes les cérémonies non disponibles en ligne. Mais l'avantage d'une telle méthode est vite apparu supérieur aux inconvénients: l'accroissement considérable du nombre de cas empiriques à disposition.

Au-delà des moteurs de recherche vidéo connus, mes explorations se sont orientées dans trois directions: des sites de dépôt de vidéos en ligne (Dailymotion ou Youtube), des sites de joueurs (judgehype ou jeux online.info), et les sites de guildes. Enfin, même si certains exemples provenaient du monde nordaméricain, je me suis volontairement limité au monde francophone et de ce fait aux serveurs en langue française de WoW. II s'agit 


\section{Olivier SERVAIS}

d'abord des serveurs officiels de la société Blizzard. Mais les vidéos récoltées par ce processus de recherche m'ont amené rapidement à y adjoindre les serveurs privés.

Sur cette base, 141 traces vidéos francophones de cérémonies de mariage ont été recueillies. De ce nombre, j'ai retranché celles qui se sont avérées manifestement illisibles ou ne permettaient pas un apport d'information minimal.

À cet égard, la nature du serveur (privé ou officiel ; jeu de rôle ou normal; PVE ou $P V P^{6}$ ), l'identité des collectifs impliqués (Guilde ou autres) ou la capacité à identifier les protagonistes (officiants, mariés, témoins, public) sont apparus comme des données essentielles afin de pouvoir par la suite récolter des informations complémentaires (forum, site de guilde, contact direct avec des participants). Ces éléments supplémentaires devaient permettre une contextualisation plus fine des données vidéos audelà de ce que les images et les sons nous indiquent en première vue. Au final, c'est donc sur 100 séquences analysées que se basent les informations qui vont suivre.

À partir de ce stock de données, j'ai alors mené systématiquement une mini-ethnographie de la vidéo en question pour recontextualiser ce qui se donnait à voir à l'écran. Ce travail s'est pratiqué via les forums où avaient été postées les vidéos, via le site des guildes concernées, par contacts directs avec les protagonistes identifiés, ou tout autre méthode susceptible de donner de la chair à la structure de base vue en vidéo.

\footnotetext{
${ }^{6}$ PVE signifie un style de serveur favorisant les combats contre les agents informatiques mus par le programme; PVP les serveurs orientés affrontement entre avatars et donc entre joueurs.

tic\&société - 9 (1-2), 2015
} 
Cérémonies de mariage dans World of Warcraft

\section{Constats empiriques}

En l'état de la recherche, que nous disent ces cérémonies ?

Premier constat, les serveurs où se sont déroulées ces solennités sont multiples. Ces 100 séquences filmées ont été recensées sur plus de 29 serveurs différents. Numériquement faibles, les mariages n'en demeurent pas moins une pratique étendue, et cela bien au-delà des seuls "Royaumes » du jeu de rôle. Dix serveurs privés font partie de cet inventaire et témoignent de plus de créativité dans l'élaboration des décors, notamment grâce au crafting - façonnage informatique - possible du lieu de cérémonie. Ces serveurs se distinguent assurément par la qualité des ornements et des habillements.

Où s'organisent les cérémonies ? 43 sur 100 dans la cathédrale de Hurlevent, la capitale des humains de l'Alliance. En dehors de cet espace dominant, c'est la diversité qui prévaut : 35 autres lieux. Le lieu suivant en termes d'usage est le temple d'Elune à la cité elfe de Darnassus avec 10 cérémonies. II n'y a pas de corrélation entre la nature des avatars-mariés et le lieu des cérémonies.

Sans surprise, c'est du côté de la Horde que la diversité affole la boussole. Sur 23 cérémonies de la Horde, on trouve 20 lieux différents. L'absence d'un édifice à morphologie religieuse évidente et situé centralement dans cette faction entraîne ainsi une diversification des investissements de l'espace - là où, pour l'Alliance, la cathédrale de Hurlevent s'impose comme une évidence à tous, et parfois le temple de Darnassus. Le poids des déterminants techniques, l'existence d'un espace reconnu comme religieux et proche des lieux de rassemblements, sont dans ce cas très importants. Par ce choix préférentiel, le mariage semble donc bien, du point de vue de l'imaginaire des joueurs, un acte religieux.

D'ailleurs la nature de l'officiant va dans la même direction. Car qui préside les cérémonies? Un personnage religieux. De fait, la diversité des lieux fait place à une forte homogénéité des célébrants, et une homologie entre factions. Dans pratiquement tous les cas de figures, l'officiant est un prêtre (ou apparenté, shaman, démoniste). Il est essentiellement seul, même si plusieurs 


\section{Olivier SERVAIS}

cérémonies sont animées à deux ou trois. Dans bien des cas ce "prêtre " est membre d'une des guildes investies dans la cérémonie. De rares occasions voient un non-prêtre présider, dont un maître de guilde ou un ami proche. La symbolique de l'avatar prime ainsi sur la relation aux joueurs, même si les deux dimensions peuvent s'articuler. En effet, il faut se méfier de conclure hâtivement à une banalité déconcertante. Les trajectoires des mariés et de l'officiant restent très personnelles au-delà des formes. Il est capital de rentrer dans les significations. Le cas de Yeshua, un nain vivant dans le Royaume "Conseil de l'ombre ". et marié à Calinae le 5 août 2012 dans les jardins de Hurlevent ${ }^{\dagger}$ est emblématique. Tous deux viennent de la même guilde, Rage, et la vidéo des festivités est prise par Khyarä. Et deux mois plus tard, le 20 octobre 2012, le marié Yeshua officie pour le mariage de cette même Khyarä avec Regex dans un temple de Pandarie aux allures bouddhiques ${ }^{8}$. Ici le montage est de la mariée ellemême. Cette logique réticulaire des relations qui président au mariage est symptomatique de cette logique d'autonomie des cérémonies. Les réseaux de proximité priment et les engagements et célébrations se construisent au jour le jour à partir d'initiatives individuelles. Ainsi, les cérémonies s'y bricolent selon les compétences disponibles parmi l'entourage de jeu et sur la base d'un réseau affinitaire d'amis, dont au premier chef les camarades de guilde.

La nature des unions demeure assez traditionnelle : les avatars mariés le sont essentiellement à deux, hétérosexuels et de même faction. Les exceptions à ce schéma sont peu nombreuses. Si l'essentiel des mariages sont internes à une même faction, 53 sur 100 se déroulent entre avatars de même race. Soit un relatif équilibre. La faction fonctionne ainsi bien plus comme une « race » que les types d'avatars.

Enfin, sur 78 mariages où les guildes sont identifiées, 58 sont intra-guildes, soit une large majorité. On peut qualifier dès lors

\footnotetext{
$7<$ https://www.youtube.com/watch?v=RsvdhzheHi4>, dernière consultation le 16 avril 2015.

$8<$ http://www.youtube.com/watch? $v=C 8 u U O B F L C H s>$, dernière consultation le 16 avril 2015.

tic\&société - 9 (1-2), 2015 


\section{Cérémonies de mariage dans World of Warcraft}

sans conteste ces mariages d'endogames au sein de la famille affinitaire que constitue de fait la guilde. Seules 5 cérémonies sont ouvertement interguildes. Une seule se revendique d'une union entre guildes. Dans ces réjouissances, c'est donc bien avant tout la guilde qui œuvre. Cette activité ne lui rapporte rien directement en termes de jeu, si ce n'est un moment de jeu de rôle, mais elle contribue aussi à unir ses membres autour d'une activité symbolique et ludique commune.

Le public joueur présent est aussi très variable. Les futurs conjoints disposent majoritairement de 2 à 4 témoins. Seuls 17 évènements sont sans témoins privilégiés. L'assistance s'étale d'aucun à 40 personnages. La moyenne se situe aux environs de 13. Seules 18 cérémonies atteignent 20 spectateurs et plus. Cela correspond aux critères de taille d'une petite guilde, mais celle qui est la plus courante (Williams et al., 2006). Les cérémonies apparaissent ainsi comme des évènements intimes, même si seules trois cérémonies se déroulent en solitaire, réunissant uniquement les mariés et l'officiant. Les mariages comportent en effet - et ce de manière centrale - une dimension sociale, on célèbre avec d'autres. La dimension relationnelle du rituel est donc essentielle. On peut même dire que la cérémonie est d'abord un événement de sociabilité où l'on réunit autour des futurs conjoints son réseau de proches virtuels, bien souvent sa guilde.

Le rituel en lui-même apparaît ainsi moins central que la célébration au sens plus large. L'événement social et relationnel prime très certainement sur la forme rituelle. Sur le plan des signes d'ailleurs il est difficile de tirer de multiples conclusions, si ce n'est un élément qui frappe l'observateur: toutes les cérémonies font l'objet d'un accoutrement particulier. Un code vestimentaire implicite prévaut. La mariée est souvent en blanc, le marié est costumé et l'assemblée bien habillée. Les équipements guerriers et autres attirails habituels ont été rangés au placard au profit d'habillements de fête ou à tout le moins originaux. Des titres et surnoms apparaissent aussi spécifiquement pour ce jour. Cette distinction par l'habit est généralisée et témoigne d'une césure corporelle avec le temps normal. Le temps du rite s'inscrit dans l'apparence des avatars. C'est probablement le seul comportement assumé par tous. 


\section{Olivier SERVAIS}

Si l'on s'arrête sur les positions spatiales des acteurs de la cérémonie, on constate une diversité des configurations. L'adaptation au lieu semble prévaloir, mais comme un endroit s'impose, côté Alliance, il limite les possibles. Hors de la cathédrale de Hurlevent une très grande diversité prévaut. Les cérémonies en pleine nature sont à cet égard très novatrices et font preuve d'un effort de personnalisation important. Selon que l'on soit dans un serveur privé ou non, la créativité et le façonnage des décors pourront trouver plus ou moins à s'exprimer.

Une séquence rituelle semble ainsi s'imposer partout : arrivée des conjoints (avec souvent une haie d'honneur), moment autour de l'autel ou de l'officiant, échange des consentements, cris de joie du public. Cette séquence peut inclure également des feux d'artifice, l'usage de spots de lumière ou différentes danses. On retrouve donc la séquence rituelle évoquée par Rakke-Antweiler (2007), mais de manière beaucoup moins détaillée. C'est, au fond, comme si le détail de la majorité des rituels comptait peu. Les discours des officiants sont relativement stéréotypés et n'apportent finalement que peu d'information. Ils sont centrés sur l'échange des consentements qui s'avère constituer le cœur du rite. Notre source principale, les vidéos, nous donne hélas peu d'éléments sur l'avant cérémonie, même si les quelques cas montrent de longs temps informels d'attente collective et de préparation. L'après est nettement plus convenu : on fait la fête ! Et ce moment revêt manifestement une importance cruciale vu le temps qui y est consacré et les changements, notamment d'habillement, que ce moment génère. II n'est pas rare que les convives se dévêtissent durant la fête et dansent un très long moment. Lorsque l'activité se déroule à Hurlevent, c'est assez systématiquement dans une taverne que la fête s'organise, avec danses endiablées, jeux de lumière, dénudement et alcool. Ailleurs la nature des fêtes est plus variable, mais semble similairement importante pour les participants. On a plusieurs cas où la fête se déroule directement sur le lieu de cérémonie, sans véritable césure spatiale. Mais cette perspective demeure très minoritaire, et l'on perçoit régulièrement ce besoin de segmenter un lieu rituel (sacré) et la fête profane. D'ailleurs, on peut souvent distinguer le rite du reste de la noce par le ton sérieux du moment rituel. L'utilisation de l'humour n'est pas 
Cérémonies de mariage dans World of Warcraft

fréquente et l'usage de l'argot des joueurs limité lors de ces moments solennels.

\section{Normalisation des cérémonies}

En passant en revue le portrait type d'un mariage sur WoW, on est d'emblée frappé par la forme convenue et le scénario relativement classique de la majorité des célébrations. Certes, il existe une différence limpide entre Alliance et Horde et l'uniformité du modèle est avant tout présente chez la première. De fait, de la multiplicité des lieux utilisés par la Horde pour célébrer ces noces engendre clairement un foisonnement d'inventivité pour choisir et adapter un espace numérique au projet de cérémonie. Je songe, par exemple, à plusieurs cas où les guildes concernées ont intégré dans la cérémonie l'étape de purification des lieux qui consistait à éliminer les agents ennemis gardant le lieu et mus par l'ordinateur, avant de pouvoir commencer plus classiquement les célébrations.

Mais au-delà de ces distinctions, les cérémonies, où qu'elles se déroulent, demeurent relativement classiques, et se référent souvent explicitement au modèle de mariage à l'occidental, articulant ritualités séculières (haies d'honneur, fêtes, feux d'artifices, etc.) et cérémonies religieuses (prêtres, robes blanches, consentements). II n'en demeure pas moins que des spécificités peuvent être repérées même dans les cérémonies aux allures les plus convenues (Boutet, 2012).

Dans la plupart des cas, les guildes interviennent dans une fonction d'adaptation et d'organisation d'événements dans lesquels elles espèrent implicitement pouvoir traduire une partie de leurs valeurs. Ces mises en œuvre orientent l'esprit des activités et donnent une touche particulière aux célébrations.

Ainsi, par exemple, au sein d'une de ces guildes francophones, "l'alliance des Seigneurs " ${ }^{9}$, deux avatars se sont mariés, et les membres du collectif ont organisé virtuellement la célébration.

\footnotetext{
${ }^{9}<$ http://forum.seigneurs.org/>, dernière consultation le 16 avril 2015.
} 


\section{Olivier SERVAIS}

Dans le présent cas, ils se sont rendus à la cathédrale au cœur de Hurlevent. Le maître de guilde préside la cérémonie d'engagement, première spécificité, et à la sortie les mariés sont salués par l'ensemble du groupe agenouillé, deuxième patte originale. Certes la guilde est limitée par les contraintes de l'ordinateur. II n'y a pas de possibilité avec le logiciel de lancer du riz ou d'autres choses, les joueurs créent donc avec les contraintes que leur impose la technique. Dans ce cas précis, la guilde va saluer les mariés en se plaçant en deux haies d'honneur parallèles au sortir de l'édifice. À l'image de la Guilde, le mariage va travailler la forme. L'Alliance des seigneurs va tout d'abord créer une histoire, va se raconter elle-même à travers l'événement. Ici le mariage est directement lié à la création de la guilde. On institue un collectif de jeu, dans un esprit de jeu de rôle, et d'emblée on célèbre ensemble le mariage de deux d'entre eux.

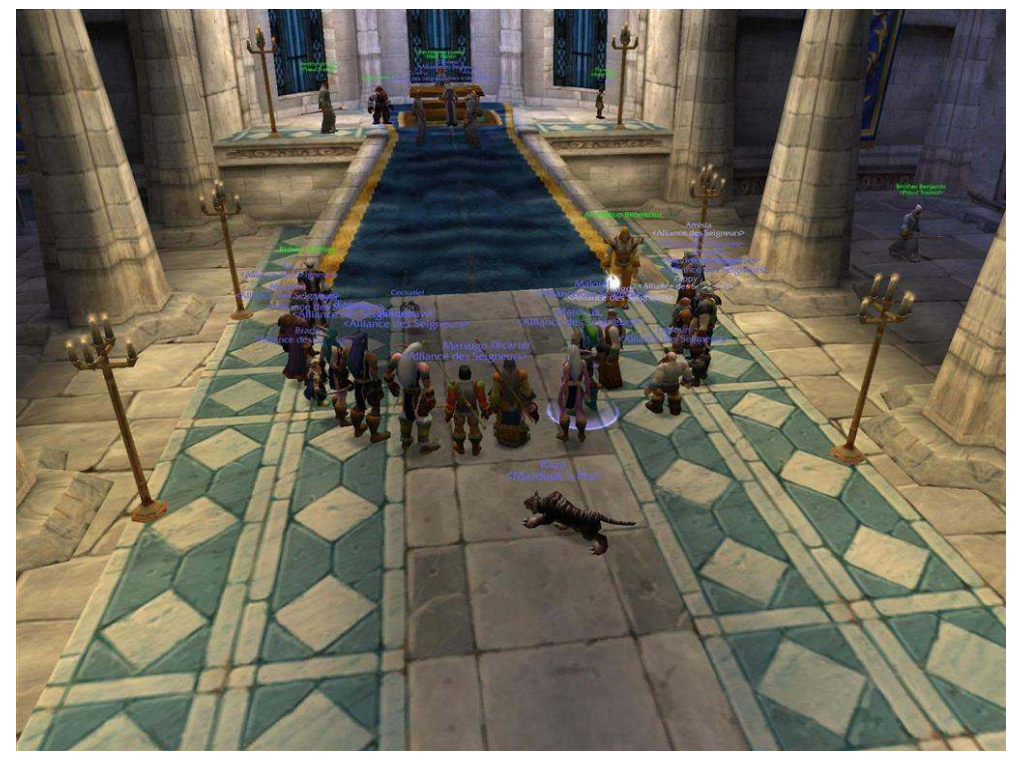

Fig. 1. Célébration du mariage de l'Alliance des seigneurs dans la cathédrale du Hurlevent. Source : <http://forum.seigneurs.org/>, dernière consultation le 16 avril 2015. 
Cérémonies de mariage dans World of Warcraft

"Laissez-moi vous conter l'histoire du premier rassemblement de l'Alliance des Seigneurs... Gnomes, Nains, Humains et Elfes s'étaient donné rendez-vous à Stormwind-la-Grande afin de montrer au peuple d'Azeroth ce que représentait être un Seigneur... L'entrée de Stormwind voyait arriver petit à petit chacun et la joie de tous de se retrouver était immense... $\gg .{ }^{10}$

La guilde fait sa «joyeuse entrée » à Hurlevent, elle confectionne collectivement son tabard de guilde (survêtement placé au-dessus de l'armure et qui présente les armoiries de la guilde). Une fois le collectif identifié de la sorte, ses membres se rendent immédiatement à la cathédrale pour y célébrer un mariage. Une haie d'honneur clôt les cérémonies et lui fait place un moment de fête. Si la séquence n'a rien d'inédit, le soin à lier narrativement le mariage et la vie de la guilde est une véritable particularité. Le mariage est explicitement signalé comme un événement du collectif. II entre complètement dans la vie de la communauté. D'ailleurs, 22 membres sur 22 étaient présents, une audience qui témoigne de l'importance du moment: une symbolique instauratrice du collectif. L'ensemble de ce moment apparaît très codé, tout de cette configuration de mariage témoigne d'un style à la fois " jeu de rôle ", où la narration fictionnelle est prise très au sérieux, et, parallèlement, d'une prégnance du collectif sur ses membres. L'importance de la guilde s'en ressent de bout en bout. II est d'ailleurs frappant de voir que si les cérémonies humoristiques existent, elles sont loin d'être majoritaires. Durant le moment rituel, le sérieux prévaut. D'où mon hésitation à rattacher ces célébrations à du carnavalesque et du faire-semblant (Berry, 2012, p.162-173).

D'autres cas s'apparentent à celui-ci. Parfois symboliquement, c'est le chef de guilde lui-même qui est un des deux époux. ${ }^{11}$ Ainsi en est-il le 18 février 2012, lorsque Blackmämba et Kerkov sont

\footnotetext{
${ }_{10}^{10}<$ http://seigneurs.org/codes/>, version du mercredi 17 octobre 2007.

${ }_{11}^{11}<$ https://www.youtube.com/watch?v=q2XpbjJQyzU $>$, dernière consultation le 16 avril 2015.
} 


\section{Olivier SERVAIS}

unis au sein de la Guilde des Zelfiques Heureux, dont Kerkov est le chef d'environ 200 membres.

Mais des configurations plus inattendues, voire carrément marginales, existent aussi. Je songe ici notamment à des guildes d'une faction réunissant leurs ouailles en zone neutre (Dalaran, Cabestan ou Baie au butin) afin d'organiser des cérémonies interfactions. Parfois, la collaboration entre factions va assez loin, ces moments marquant alors une véritable trêve dans le jeu. Les mariages à Hurlevent pour deux joueurs de la Horde constituent un bon exemple de ce type de paix de Dieu. En mai 2012, sur le serveur Ner'zhul ${ }^{12}$, Cornedure, maître de la guilde "Sentence », a participé à l'organisation du mariage en jeu de deux de ses membres : Chainsäw et Flia, deux demoiselles. L'évènement a été rejoint par quelques joueurs Hordeux, mais aussi par des membres de l'Alliance qui ont accepté le jeu de la trêve ${ }^{13}$.

Mais pourquoi ces cas restent-ils minoritaires, particulièrement dans l'Alliance? Je retiendrais deux éléments parmi d'autres permettant de comprendre cette distinction entre homogénéité conformiste de l'Alliance et diversité contrainte de la Horde. II semble assez évident qu'une combinaison entre affordance des lieux - c'est-à-dire contrainte par le dispositif technique d'une part (Berry, 2012, pp.72-73) - et l'existence dans le jeu, ou dans ses alentours, de processus de normalisation d'autre part, opère en partie ce travail de régulation et d'uniformisation. De fait, comme peu de gens participent à ce type d'évènements, on ne peut pas chercher uniquement en ceux-ci la dynamique de création de la norme. Si les contraintes des lieux ont été évoquées, on peut en dire plus sur les processus de normalisation.

Deux figures de cette normalisation semblent incontournables: les forums de joueurs et les organisateurs d'events. Les forums de

\footnotetext{
${ }^{12}<$ http://worldofwarcraft.judgehype.com/news/mariage-a-hurlevent-pour-deux-joueursde-la-horde-sur-ner-zhul/s, dernière consultation le 16 avril 2015; $<$ http://sentence.fr/print.php?news.106>, dernière consultation le 16 avril 2015. ${ }^{13}<$ http://worldofwarcraft.judgehype.com/image/300951/\#img $>$, dernière consultation le 16 avril 2015.

tic\&société - 9 (1-2), 2015 


\section{Cérémonies de mariage dans World of Warcraft}

joueurs vont fournir des réponses massives et très cadrées sur ce qu'est un mariage In Game et comment il doit s'organiser. À bien des moments, on y a recours pour un coup de main technique ou une aide plus stratégique. $Y$ afficher dès lors des indications prescriptives sur les mariages renforce sans conteste le modèle dominant. II est étonnant de constater que loin d'être des lieux de créativité, ces lieux de discussion en ligne ancrent le modèle de référence.

À côté des forums, de véritables entrepreneurs d'évènements voient aussi le jour dans le jeu lui-même, et une de leurs spécialités est incontestablement la préparation de mariages. Réalisation de faire-part, planification et mise en œuvre des cérémonies, fournitures diverses dont les tenues du jour sont autant de services rendus contre pièces d'or virtuelles sonnantes et trébuchantes, ou parfois à titre gracieux.

Supernovache, une druidesse tauren, agit au sein d'un petit collectif, la team G.O, créée avec un autre joueur Lenwë. Elle s'est ainsi spécialisée dans l'animation en jeu sur le serveur Sargeras, côté Horde. Outre des animations ludiques collectives atteignant jusqu'à plusieurs centaines de participants, elle s'est focalisée sur les mariages dans le jeu. Elle offre costumes, prêtre, lumière, publicité, photos souvenirs, etc. Et même si cette entreprise individuelle tient en bonne partie lieu de fiction, insérée dans le ludisme du jeu, il n'en demeure pas moins que ce type d'activité diffuse elle aussi, de cette façon, un modèle très normé.

Organisez vos mariages sur Sargeras grâce à "Dis moi oui l"

Par Maitre Crieur * 13 Mar 2012, 13:34 * sargeras, mariage, dis mol oul

Vous avez envie d'amour et d'un peu plus que de l'eau fraîche?

Vous désirez organiser une somptueuse réception de l'ambassadeur pour un mariage avec des feux d'artifices par myriade?

Alors faite confiance à Supernovache et son équipe pour vous aider à réaliser le plus beau mariage possible sur le royaume Sargeras !

En choisissant l'agence "Dis moi oui", vous profitez:

- de création des costumes, d'une fabrication des bagues, et d'un fleuriste pour la décoration

- d'un prêtre mit à disposition pour célébrer la cérémonie

- des lumières par milliers de quoi éclairer les mariés!

- d'une communication sur l'événement, envoie des invitations... tout le monde sera au courant pour votre

grand jour !

- d'un album photo pour que ce souvenir soit éternel pour la fin du mariage <3 
Olivier SERVAIS

Fig.2. Publicité pour les activités de Supernovache. Source : <http://www.warcraftpeople.com/projets-d-evenements/organisez-vosmariages-sur-sargeras-grace-a-dis-moi-oui-t632.html>, dernière consultation le 16 avril 2015.

\section{Conclusion : ritualités religieuses numériques et rapports politico-religieux}

Que conclure de ce parcours au pays des noces francophones d'Azeroth ?

1. Sur le transfert rituel d'abord. Pour rappel la théorie reliait trois éléments: la transformation sous de multiples formes du rituel, l'invention de nouveaux éléments relatifs au nouvel univers de réception et enfin l'exclusion de parties du rituel originel.

Une séquence rituelle se dégage qui rencontre globalement celle de Rakke-Antweiler (2007), particulièrement du côté de l'Alliance où les cérémonies sont ancrées dans un édifice unique pour $75 \%$ des solennités. Cette séquence est le produit d'un transfert de formes rituelles morphologiquement religieuses en Europe occidentale, vers une cérémonie en ligne. Si les séquences rituelles peuvent être importantes, les détails des rites en eux-mêmes sont relativement limités, très normés, bref traduisent un «prêt-à-porter rituel » souvent imposé par les limites des ressources graphiques disponibles dans ces univers numériques, mais surtout véhiculé par des forums et des acteurs spécialisés. Des exceptions notoires ressortent dans deux champs qu'il faudra investiguer plus en profondeur par la suite: les serveurs privés d'une part, et les cérémonies de la Horde de l'autre. Dans le premier cas, c'est la diminution de la contrainte technique, la liberté de reprogrammer le design, qui accroît la créativité des cérémonies. Dans le second, c'est l'inexistence d'un lieu s'imposant d'évidence comme l'endroit où célébrer qui ouvre les possibilités. On peut parler, plus généralement, d'une marge 


\section{Cérémonies de mariage dans World of Warcraft}

qui permet au bricoleur de jouer davantage avec le rite et la cérémonie (Hamayon, 2012, pp.301-303).

Dans tous les cas, ces ritualités numériques contiennent un grand nombre de références très personnelles liées à l'itinéraire des époux ou de la guilde, exactement comme on le voit dans l'évolution des cérémonies de mariage hors ligne aujourd'hui. De fait, nos observations des mariages virtuels nous amènent à conclure que ces cérémonies semblent un bon témoignage du bricolage contemporain de ce type de rituel (Segalen, 2005, pp.161-163). Bien entendu le contexte y est spécifique, particulièrement dans les contraintes techniques et spatiales.

2. La problématique du «transfert rituel », que nous avons évoquée auparavant, se focalise sur le rite en tant que tel et a dès lors tendance à "ritualiser » ces cérémonies. Mais lorsque l'on observe ces "rites", ceux-ci semblent marginaux au sein de la séquence cérémonielle. II y a manifestement un rite, mais il est très sommaire et dépouillé et, à l'exception du temps du consentement, il déploie peu de signification explicite.

Pour reprendre Houseman, "Certains rites sont très largement composés de formules liturgiques. Toutefois, de façon générale, ce qui compte est moins le contenu sémantique de tels énoncés [...] que l'acte même de les proférer. " (Houseman, 2008, p.1), et je rajouterai celui qui les profère, et les personnes devant lesquelles il procède.

En outre, l'avant et l'après du rite prennent une place sensible. On leur consacre largement plus de temps que le rite en lui-même. Nous pensons de ce fait que l'important n'est pas d'abord le rite et sa symbolique, mais la cérémonie globale et ce qu'elle permet au collectif : se rassembler, inventer ensemble un rite, célébrer et fêter collectivement, bref exprimer l'existence d'un groupe à partir d'une créativité communautaire. Qu'elles soient analogiquement (Servais, 2013) ou explicitement religieuses, ces célébrations revêtent une fonction symbolique centrale au sein d'un collectif ou d'un réseau d'interconnaissances virtuels. Cette seconde dimension manifeste selon nous une performance collective. Selon la nature de l'investissement dans un collectif virtuel institué ou 


\section{Olivier SERVAIS}

non, la cérémonie renforcera le réseau de liens personnels ou, dans les cas les plus communautaires, participera à l'institution imaginaire du collectif de joueurs (pour paraphraser Castoriadis, 1975). II participe à élaborer le noyau imaginaire, les récits et histoires partagés, qui constitueront l'identité du collectif et entretiendront le sentiment d'appartenance au groupe.

3. Cette hypothèse prend tout son sens à la lumière des acquis de la recherche sur la vie des guildes. Celles-ci apparaissent sans conteste des communautés de pratiques, se construisant par l'expérience partagée (Berry 2007, p.83). En ce sens elles sont aussi un lieu de sociabilité. Williams et al. (2006) ont montré que $60 \%$ des joueurs interrogés qualifient leur guilde d'un caractère social, c'est-à-dire ayant pour premier but l'interaction sociale avec les autres joueurs, et même pour des groupes experts, délassement et camaraderie demeurent centraux (Chen, 2012, pp.55-82). Les variations se dessinent plutôt selon l'échelle de la guilde. Petite horizontale et soudée comme la Communitas de Turner (1967, p.97), versus plus grande requérant organisation, hiérarchie et structure. La balance de l'une à l'autre s'opère autour des 35 membres. Mais les petits rassemblements sont freinés par leur taille, et donc leur fragilité démographique. Les guildes ont dès lors tendance à vouloir grandir (Ducheneaut et al., 2007). De ce fait, elles sont contraintes à s'organiser pour survivre. Elles institutionnalisent chartes d'objectifs et de comportement des membres, processus de recrutement et site web. A travers ce double portrait contrasté, les chercheurs cités confirment que les guildes, quelle que soit leur taille, sont des entités fragiles (Williams et al., 2006), tandis que la qualité du leadership est avancée comme le meilleur frein à la désintégration d'une guilde.

4. Dans la version la plus communautaire des mariages décrits, ceux de guilde, le comportement des joueurs renforce les thèses de Godelier (2007). De fait, comme j'ai essayé de le démontrer, ceux-ci favorisent la perpétuation du collectif qu'est la guilde, son imaginaire, ses règles et ses hiérarchies. La guilde joue en quelque sorte le rôle d'une famille élargie, d'un lignage ou d'une structure pour reprendre Turner (1969, pp.97-98). A cela près que la guilde se trouve plongée dans des rapports techniques (game design) qu'elle complète ou subvertit par l'institution d'un cadre 
Cérémonies de mariage dans World of Warcraft

politico-religieux propre. L'inclusion dans ce collectif est ainsi conditionnée par la participation à une alliance politico-religieuse entre ses membres. La charte de guilde en est l'expression symbolique, la définition substantielle du fameux rapport politicoreligieux dont parle Maurice Godelier. Qu'en est-il alors du mariage ? La dimension endogamique de la cérémonie permet l'expérience collective d'un esprit, et, derrière lui, de valeurs de la guilde. Le mariage est de guilde, organisé par ses membres. De ce fait, il symbolise et actualise l'alliance du collectif. Cependant, à de rares exceptions, il ne met pas en scène autre chose que le pouvoir de ses membres d'être présents et du collectif de se rassembler et de rendre possible l'évènement.

Dans un univers où les joueurs sont très autonomes, et où d'un « click » ils peuvent se déguilder, c'est-à-dire quitter le collectif, il s'agit d'une modalité politico-religieuse, qui consiste à renforcer le sentiment d'une appartenance structurellement précaire.

\section{Références bibliographiques}

BAINBRIDGE W.S., 2010, Online Worlds: Convergence of the Real and the Virtual, Artington, Guildford, Springer.

BAINBRIDGE W.S., 2010, The Warcraft civilization. Social science in a virtual world, Cambridge, MIT Press.

BAINBRIDGE W.S., 2013, eGods. Faith fantasy in Computer Gaming, Oxford, Oxford University Press.

BERRY V., 2007, "Les Guildes de joueurs dans l'univers de Dark Age of Camelot : apprentissages et transmissions de savoirs dans un monde virtuel ", Revue française de pédagogie, $n^{\circ} 160$, juillet-septembre. <http://rfp.revues.org/738>, dernière consultation le 16 avril 2015.

BERRY V., 2012, L'expérience virtuelle. Jouer, vivre, apprendre dans un jeu vidéo, Rennes, Presses universitaires de Rennes. 


\section{Olivier SERVAIS}

BOELLSTORFF T., 2008, Coming of Age in Second Life. Princeton, Princeton University Press.

BOELLSTORFF T., 2009, "A Typology of Ethnographic Scales for Virtual Worlds", dans W. S. BAINBRIDGE (dir), Online Worlds: Convergence of the Real and the Virtual, London, Springer, pp.123-134.

BOUTET M., 2012, « Jouer aux jeux vidéo avec style. Pour une ethnographie des sociabilités vidéoludiques ", Réseaux, $\mathrm{n}^{\text {os }} 173$ 174, pp.207-234.

BRIGNALL T., 2008, "Guild life in the world of Warcraft : Online game tribalism », dans T. ADAMS, S. SMITH, Electronic tribes: The virtual world of geeks, gamers, shamans, and scammers, Austin, University of Texas Press, pp.110-123.

CASTORIADIS C., 1975, L'Institution imaginaire de la société, Paris, Points.

CERTEAU M. de, 1990, L'invention du quotidien, 1. Arts de faire, Paris, Gallimard.

CHEN M., 2012, Leet Noobs. The Life and Death of An Expert Player Group in World of Warcraft, New-York, Peter Lang.

CHRISTIANS L.-L., SERVAIS O. (dir), 2005, «Religious Bricolage? How Do We Conceptualize the 'Mixture' of Religions ? », Social Compass, vol.52-3, pp.275-279.

DUCHENEAUT N., YEE N., NICKELL E., MOORE R.J., 2006, "'Alone together?' Exploring the social dynamics of massively multiplayer online games", dans Proceedings of the SIGCHI Conference on Human Factors in computing systems. New-York, Association for Computing Machinery, pp.407-416.

DUCHENEAUT N., YEE N., NICKELL E., MOORE R.J., 2007, "The Life and Death of Online Gaming Communities : A Look at Guilds in World of Warcraft ", dans Proceedings of the SIGCHI Conference on Human Factors in computing systems, New York, Association for Computing Machinery, pp.839-848.

DUFOUR S. et BOUTAUD J.-J., 2013, « Extension du domaine du sacré ", Questions de communication, vol.23, pp.7-30.

tic\&société - 9 (1-2), 2015 
Cérémonies de mariage dans World of Warcraft

GODELIER, M., 2007, Au fondement des sociétés humaines. Ce que nous apprend l'anthropologie, Paris, Albin Michel.

HAMAYON R., 2012, Jouer. Une étude anthropologique, Paris, La Découverte.

HEIDBRINK S., 2007, "Exploring the Religious Framework of the Digital Realm. Offline-Online-Offline Transfers of Ritual Performance ", Masaryk University Journal of Law and Technology, vol.1, n², pp.175-184.

HEIDBRINK S., MICZEK N., RADDE-ANTWEILER K., 2011, "Contested Rituals in Virtual Worlds » dans R. GRIMES et al. (dir), Ritual, media, and Conflict. Oxford, New-York, Oxford University Press, pp.165-187.

HELLAND C., 2013, « Ritual », dans H. CAMPBELL (dir), Digital Religion. Understanding religious practice in new media worlds, Londres-New York, Routledge, pp.25-40.

HOUSEMAN M., 2008, «Relations rituelles et recontextualisation ", dans $\mathrm{H}$. WAZAKI (dir), Multiplicity of meaning and the Interrelationship of the Subject and the Object in Ritual and Body texts, Nagoya, Nagoya University, pp.109-114.

HOUSEMAN M., SEVERI C., 1998, Naven or the Other Self. A Relational Approach to Ritual Action [1994], Leiden, Brill Publications.

KLINK M. L., 2008, I Type the Amens and Think the Rest: An Ethnographic Look at Religion in Virtual Reality, mémoire de Bachelor, Reed College.

LANGER R., LÜDDECKENS D., RADDE K., SNOEK J., 2006, «Transfer of ritual », Journal of Ritual studies, $\mathrm{n}^{\circ} 20-1$, pp.1-10

MICZEK N., 2008, "Online Ritals in Virtual Worlds: Christian Online Services between Dynamics and Stability ", Online Heidelberg Journal of Religion on the Internet, vol.3, $\mathrm{n}^{0} 1$.

NARDI B., 2010, My Life as a Night Elf Priest: An Anthropological Account of the World of Warcraft, Ann Arbor, University of Michigan Press. 


\section{Olivier SERVAIS}

PERKINS A., 2011, Religious Experience in World of Warcraft, mémoire de Master, San Marcos, Texas State University.

PIETTE A., 2003, Le fait religieux. Une théorie de la religion ordinaire, Paris, Economica.

RADDE-ANTWEILER K., 2007, "Cyber-Rituals in Virtual Worlds. Wedding-Online in Second Life », Masaryk University Journal of Law and Technology, vol.1, n², pp.185-196.

RADDE-ANTWEILER K., 2010, «Wedding Design Online: Transfer and Transformation of Ritual Elements in the Context of Wedding Rituals », dans C. BROSIUS, U. HUSKEN (dir), Ritual Matters, Londres, Routledge, pp.328-353.

REID E. M., 1999, "Hierarchy and Power : Social Control in Cyberspace ", dans M.A. SMITH, P. KOLLOCK (dir), Communities in Cyberspace, Londres, Routledge, pp.107-33.

SEGALEN M., 2005, "L'invention d'une nouvelle séquence rituelle de mariage », Hermès, n43, pp.159-168.

SERVAIS O., 2012, "Autour des funérailles dans World of Warcraft. Ethnographie entre religion et mondes virtuels ", dans J.P. DELVILLE (dir), Mutations des religions et identités religieuses, Paris, Mame-Desclée, pp.231-252.

SERVAIS O., 2013, "Louvain et l'analyse du religieux. De l'ethnologie missionnaire à l'anthropologie prospective du virtuel ». Histoire, monde et cultures religieuses, vol.26, n², pp.95-108.

SHIM J., 2010, "Til Disconnection Do We Part. The initation and Wedding Rite in Second Life" ", dans C. DETWEILER (dir), Halos and avatars. Playing video games with Go, WestminsterLouisville, John Knox Press, pp.149-162.

TAYLOR T. L., 2006, Play between worlds: Exploring online game culture, Cambridge, MA, MIT Press.

TURNER V., 1990, Le phénomène rituel. Structure et contrestructure, Paris, PUF.

WEIHUA W., FORE S., WANG X., and SIK YING HO P., 2007, "Beyond Virtual Carnival and Masquerade : In-Game Marriage on 
Cérémonies de mariage dans World of Warcraft

the Chinese Internet Weihua Wu », Games and Culture, vol.2, pp.59-89.

WILLIAMS D., DUCHENEAUT N., XIONG L., ZHANG Y., YEE N., NICKELL E., 2006 «From Tree House to Barracks: The Social Life of Guilds in World of Warcraft ", Games and Culture, vol.1, pp.338-361. 